\title{
Publisher Correction: Optimal compressed representation of high throughput sequence data via light assembly
}

\author{
Antonio A. Ginart ${ }^{1}$, Joseph Hui ${ }^{2}$, Kaiyuan Zhu ${ }^{3}$, Ibrahim Numanagić ${ }^{4}$, Thomas A. Courtade ${ }^{5}$, S. Cenk Sahinalp ${ }^{3}$ \& \\ David N. Tse ${ }^{1}$
}

Correction to: Nature Communications, https://doi.org/10.1038/s41467-017-02480-6, published online 08 February 2018

The original version of this Article contained errors in the affiliations of the authors Ibrahim Numanagic and Thomas A. Courtade, which were incorrectly given as 'Department of Electrical Engineering and Computer Sciences, University of California, Berkeley, CA 94720, USA' and 'Computer Science \& Artificial Intelligence Laboratory, Massachusetts Institute of Technology, Cambridge, MA 02139, USA', respectively. Also, the hyperlink for the source code in the Data Availability section was incorrectly given as https:// github.iu.edu/kzhu/assembltrie, which links to a page that is not publicly accessible. The source code is publicly accessible at https:// github.com/kyzhu/assembltrie. Furthermore, in the PDF version of the Article, the right-hand side of Figure 3 was inadvertently cropped. These errors have now been corrected in both the PDF and HTML versions of the Article.

Published online: 26 April 2018

\begin{abstract}
(c) (i) Open Access This article is licensed under a Creative Commons Attribution 4.0 International License, which permits use, sharing, adaptation, distribution and reproduction in any medium or format, as long as you give appropriate credit to the original author(s) and the source, provide a link to the Creative Commons license, and indicate if changes were made. The images or other third party material in this article are included in the article's Creative Commons license, unless indicated otherwise in a credit line to the material. If material is not included in the article's Creative Commons license and your intended use is not permitted by statutory regulation or exceeds the permitted use, you will need to obtain permission directly from the copyright holder. To view a copy of this license, visit http://creativecommons.org/licenses/by/4.0/.
\end{abstract}

(c) The Author(s) 2018

\footnotetext{
${ }^{1}$ Department of Electrical Engineering, Stanford University, Stanford, CA 94305, USA. ${ }^{2}$ Department of Electrical Engineering \& Computer Science, Massachusetts Institute of Technology, Cambridge, MA 02139, USA. ${ }^{3}$ Department of Computer Science, Indiana University Bloomington, Bloomington IN 47405, USA. ${ }^{4}$ Computer Science \& Artificial Intelligence Laboratory, Massachusetts Institute of Technology, Cambridge, MA 02139, USA. ${ }^{5}$ Department of Electrical Engineering and Computer Sciences, University of California, Berkeley, CA 94720, USA. These authors contributed equally: Antonio A. Ginart, Joseph Hui and Kaiyuan Zhu. Correspondence and requests for materials should be addressed to K.Z. (email: kzhu@indiana.edu)

or to S.C.S. (email: cenksahi@indiana.edu)
} 